\title{
Design of the oscillating circuit in DC/DC switching power supply
}

\author{
Wei $\mathrm{Qu}^{1, \mathrm{a}}$, JingYu Sun ${ }^{1, \mathrm{~b}}$ \\ ${ }^{1}$ School of Electronic Engineering, Hilongjiang University, Harbin,Heilongjiang,150080,China \\ aaquwei70@163.com, bsjy199008@163.com,
}

Keywords: CMOS technology; switching power supply; sawtooth wave oscillator.

\begin{abstract}
The sawtooth wave oscillating circuit was proposed on order to overcoming shortcomings of traditional oscillator. This paper designed a CMOS sawtooth wave oscillator and achieved a good stability. The CMOS sawtooth wave oscillator use TennerEDA $0.25 \mu \mathrm{m}$ process library under the environment of typical application circuit loop simulation. From the experimental results: each composition can complete the corresponding circuit function, the circuit gets a stable sawtooth wave form, and oscillation frequency is $71 \mathrm{KHz}$, it can be used in DC/DC switching power supply.
\end{abstract}

\section{Introduction}

With the development of science and technology, the modern society is more and more intelligent. The development of science and technology in the field of electronic change very quickly, its application range is getting wider and wider [1]. All kinds of portable mobile power supply have become the focus of the era. Oscillation circuit as part of the main switch power supply circuit, its low power consumption not only guarantees the use of the power performance, but also prolongs the service life of the power supply. Thanks to the advantages of oscillation circuit, it ensures the quality of the switching power supply in the market competition and reduces the cost of the product. Thus, oscillation circuit quality directly influences the quality of power supply products. Therefore, we developed a stable performance of oscillation circuit is of great significance.

In this paper, we analyze the principles of oscillator and pay attention on improving the oscillator performance. This paper design of a sawtooth wave oscillator, from the experimental results shows that: sawtooth wave oscillator has good linearity, oscillation frequency is $71 \mathrm{KHz}$. It can be better applied to the switching power supply.

\section{Principle of the sawtooth wave oscillator}

Sawtooth wave oscillation circuit is composed of a phase comparator and an integral circuit. As shown in Fig.1, it is a kind of common sawtooth wave generating circuit [2,3,4]. Comparator control integrating circuit of charging and discharging, through the output of high and low points. So the circuit can cause capacitor voltage rise or fall, and change to control the output state[5,6,7]. Repeat this cycle will produce a sawtooth wave, the sawtooh wave is shown in Fig.2

\section{Design of the sawtooth wave oscillator}

In this paper, design of sawtooth wave oscillator is mainly composed of three parts: Bias circuit、 Voltage comparator、Constant current source to charge and discharge circuit.

Bias circuit. Bias circuit mainly is to provide a stable bias voltage, this circuit make the output voltage less affected by the power supply voltage. So design of bias circuit is shown in Fig.3. If MOS transistor M6 work in saturated state, when the leakage current I6 keep a certain value, the grid voltage of the M6 also keep certain values, thus, through the M5 and M6 current value is constant, so the M5 and M6 current mirror can produce a constant current, a constant current source; The same theory, the MOS transistor M7, M8, M1, M3 also constitutes the current mirror of constant current source. MOS transistor M1, M2, and M3, M4 constitute current mirror structure respectively, they also ensure the I 2 and I 3 both current value remains the same, so as to they improve the stability of the 
whole of the bias circuit, make the leakage current of the M5 equals the leakage current of M6, so that make the difference between current I5 and I6 is very small, even outside the reason to make the output and is not the same as the original, but due to the nature of circuit itself has self-regulation, it guarantees the effect of the output value of the constant. Due to M9 grid voltage is equal to the drain voltage, so M9 work in saturated zone, it makes the input output characteristic curve changes more gently.

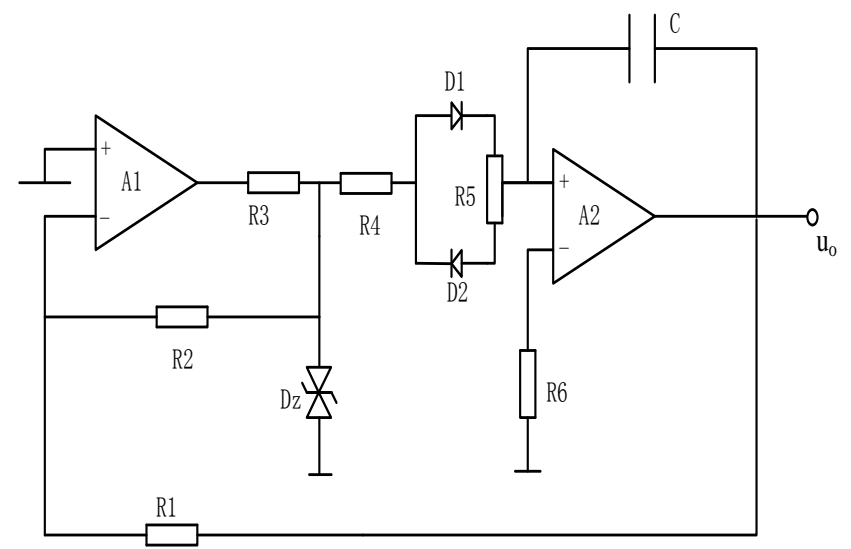

Fig.1 Sawtooth wave generating circuit

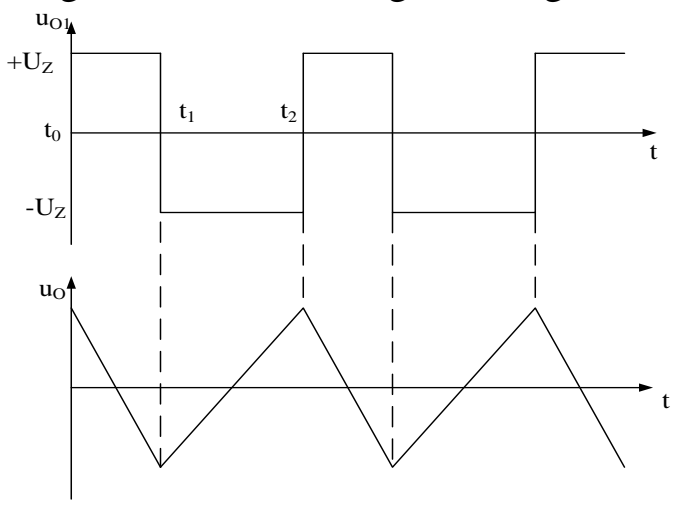

Fig.2 Waveform figure of sawtooh wave

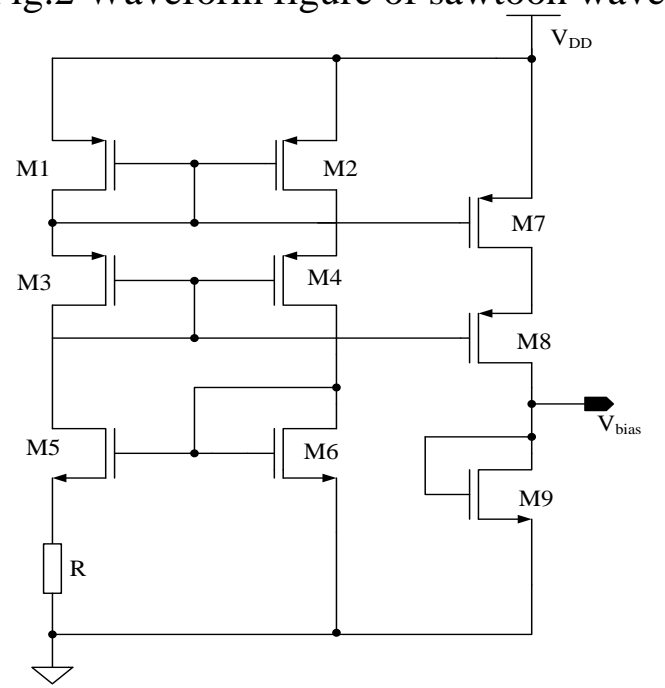

Fig.3 The Bias Circuit of sawtooth wave Oscillator

Voltage comparator. In this paper, the design of the comparator circuit adopts three-stage amplifier, the circuit principle diagram as shown in Fig.4.The first level is the differential input stage, it made the double side output into a single-ended output, M0 and M1 transistor used as current source load. The second level is CMOS current mirror structure. The third level is push-pull amplifier, which is the most simple inverter structure, because of this level as a comparator output stage, it can be output to a relatively good output. When the design, to ensure the all MOS transistor under the condition of static in the saturated zone state. When the secondary, tertiary ensure static output voltage in power 
supply voltage midpoint, it makes the third level inverter working in high gain area. Because of the comparator in the condition of open loop, you don't have to consider the frequency compensation.

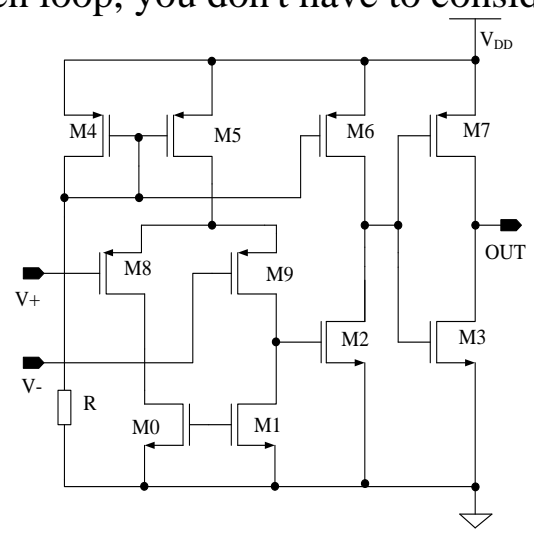

Fig.4 Voltage comparator circuit

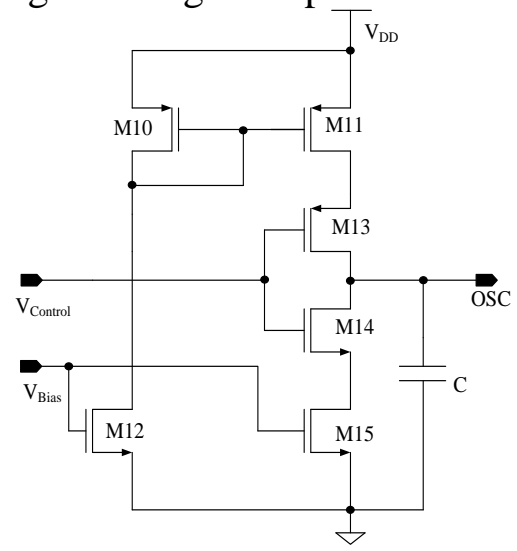

Fig. 5 Constant current source to charge and discharge circuit

Constant current source to charge and discharge circuit. According to the need to design, a constant current source to charge and discharge circuit is designed, as shown in Fig.5. The MOS transistors M10 and M12 are equivalent to a current source, and transistor M11, M13 and M14, M15 inverter structure respectively. M11, M15 and M10, M12 also are structure of current mirror. When M13 conducting, capacitance charged through M14. When M14 conducting, capacitance discharged through M13, resulting in a sawtooth wave shape

\section{Circuit simulation analysis}

Bias circuit simulation. The function of the bias circuit for the corresponding simulation analysis, the result is shown in Fig.6. As can be seen from the Fig.6, the output voltage is not along with the change of the added plus resistance voltage changes, the circuit meet the needs of design, the bias circuit can provide a stable bias voltage value.

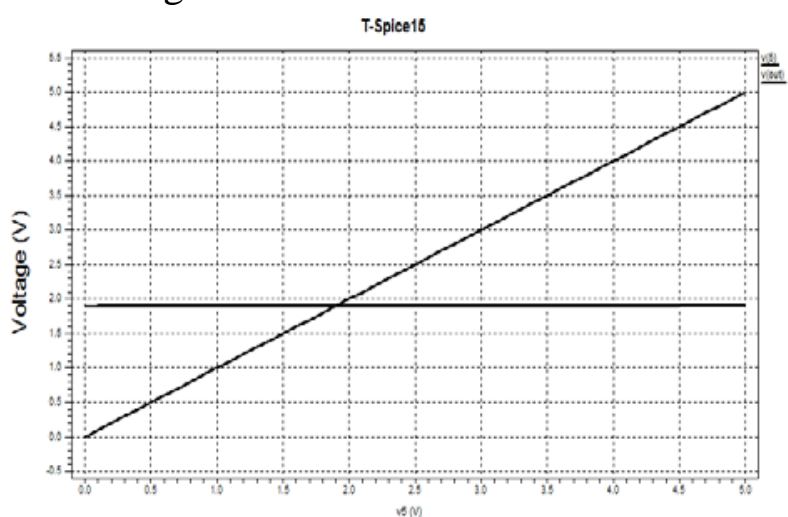

Fig.6 The function of the bias circuit simulation 
Voltage comparator simulation. As shown in Fig.7, it is the function of the voltage comparator


a low level; When $\mathrm{v}\left(\mathrm{v}^{+}\right)>\mathrm{v}(\mathrm{v}-)$, output $\mathrm{v}$ (out) is a high level. Because of the propagation delay problems, output has some delay, but the design of the comparator basically completed the need of the comparison function.

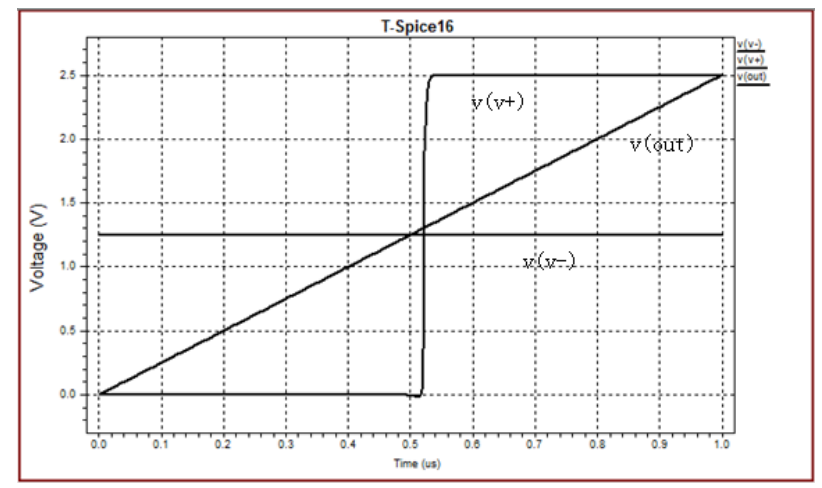

Fig.7 The function of the voltage comparator circuit simulation

Constant current source to charge and discharge circuit simulation. Through simulation, we got the simulation results are shown in Fig.8, we can be seen, and the input is low voltage, on either side of the capacitor voltage will be increased. When the input is high voltage, on either side of the capacitor voltage will be decreased. This circuit completes the function. This circuit achieves the purpose of the design.

Sawtooth wave circuit simulation. Using T-spice simulation software to oscillation circuit transient simulation, Fig.9 is the simulation results, the waveform is above voltage comparator output pulse signal, the output waveform is below the sawtooth pattern. As shown in Fig.9, we can see that at the time of the comparator output is low level, the capacitance charging. When the comparator output is high level, capacitor discharging. At the same time, according to the result of the simulation diagram, we can get the period of the oscillation circuit is $14.08 \mu \mathrm{s}$, and oscillation frequency is $71 \mathrm{KHz}$. At the same time, the power supply voltage and capacitance value can influence the change of frequency. And this circuit is controlled by a current source, so the performance of the sawtooth wave signal is good, it can satisfy the need.

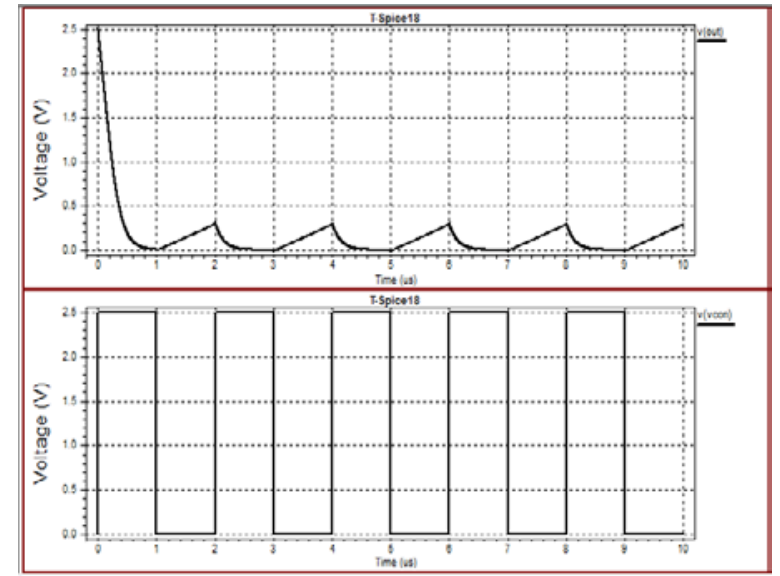

Fig.8 The function of constant current source to charge and discharge circuit simulation 

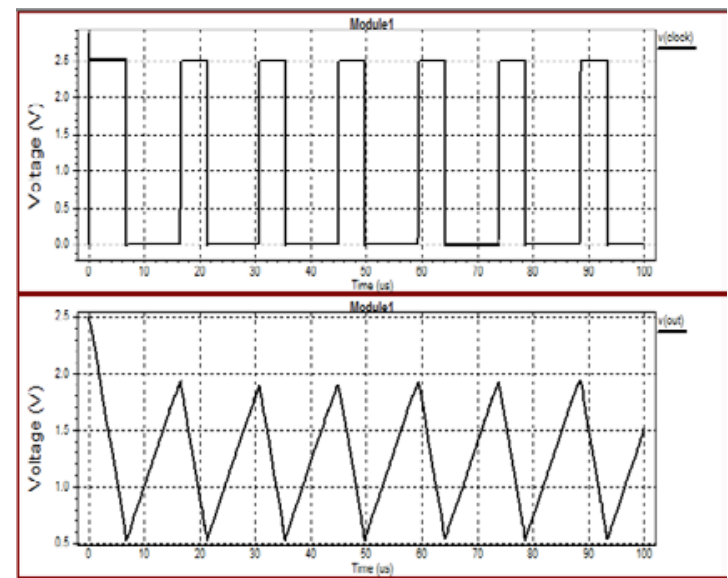

Fig.9 The sawtooth wave oscillation circuit simulation waveform

\section{Summary}

A sawtooh wave oscillator circuit design method was put forward in this article. Oscillation circuit consists of three parts: bias circuit、 voltage comparator、constant current source to charge and discharge circuit. The circuit based on TannerEDA tools, under the condition of TSMC0.25 $\mu \mathrm{m}$ process, this paper design completed the sawtooth wave oscillator, the oscillation frequency is 71 $\mathrm{KHz}$, it is suitable for the switching power supply, and it completed the design purpose.

\section{Acknowledgements}

This work was financially supported by Heilongjiang University Student Innovation and Entrepreneurship Project 2014(2014DG29).

\section{References}

[1] Xia Haisheng: A kind of applicable to DC/DC switching power supply chip sawtooth wave oscillator design, submitted to Chinese Journal of Electron Devices, vol.209-210(2009),p.567 .

[2] Van der Tweel LH, Boon P: Small signal influences upon a sawtooth oscillator, edited by Electroencephalography and clincial neurophysiology, vol.28-29(1970), p.424.

[3] Wu Qisong:A high precision CMOS weak current readout circuit, submitted to Journal of Semicondutors. vol.7-8(2009),p.61.

[4] Ma Tianhua: Design of a CMOS sawtooth wave oscillation circuit, submitted to Electronics Quaility, vol.11-14(2004),p.167.

[5] Behzad Razavi:A study of phase noise in CMOS oscillators, edtied by IEEE J.Solid-state Circuit,vol.31-32(1996),p.331.

[6] Lee Eng Han, Valerio B. Perez, Mark Lambert Cayanes:CMOS Transistor Layout KungFu. vol.56-57(2006),p.167.

[7] V.V.Dodonov:Squeezing an harmonic oscillator with a sawtooth pulse, submitted to Journal of Soviet Laser Research,,vol.14-17(1993).p.127-145. 